\title{
Design of a Novel Map POI Data Collection Model
}

\author{
Xianrong Zhang a) \\ School of Software, University of Science and Technology of China, Hefei 230000, China \\ a) Corresponding author email: zxr2016@mail.ustc.edu.cn
}

\begin{abstract}
Due to the rapid development of society and information technology, the traditional POI data collection methods can no longer meet the new requirements from users. Specifically, the data collection methods are costly, the data update speed is slow, and the attributes are not complete. Based on the geographic service interface provided by Google Maps, this paper proposes a new idea of POI data collection method and proposes a system model to implement a POI data acquisition and dynamic update prototype system. Through analysis and experiment, it is well known that This new acquisition model is very helpful for ensuring the accuracy and real-time nature of the map location.
\end{abstract}

Key words: Point of interest, data matching, spatial relations.

\section{INTRODUCTION}

POI (Point of Interest) is an abstract geographic concept, which represents the geographical entities interesting to people, such as libraries, scenic areas, mountains, and rivers [1]. POI plays an important role in various geographic information services, helping people quickly locate relevant locations. The traditional way of obtaining POI data is to allow the outsourcer to use some equipment to perform comprehensive data collection on the target area. This data collection method is high cost, low efficiency, and slow data update speed [2]. Moreover, with the continuous advancement of urbanization, the shape and position of ground buildings constantly change, which makes it more difficult to collect and update POI data.

With the popularization and development of Internet technology, users can conveniently use mobile devices to mark geographical changes that occur around them on the map. This is a quick and effective way to collect and update geographic data. This paper designs a new POI data acquisition system based on Google Maps interface and POI data collection method. With Google Maps as a unified spatial reference, users can tag geographic data they know well. At the same time, they can also judge and correct existing POI data for errors. Finally, these updated POI data will be stored in the spatial database so that it can be called by the system at the right time.

\section{LITERATURE REVIEW}

Due to the significance of POI data collection approach in academic research, a lot of scholars have studied POI data collection and updating techniques. Goodchild believes that each user is a sensor, through which they can collect the latest geographic information data, and through sharing to improve the latest situation of the entire geographical location [4]. Deren Li suggested that the use of geographical data generated by a large number of users to make up for the lack of geographic framework data, and give a wealth of details to support, so that the entire geographic system can be updated in real time [5]. Ruijia Chen uses web crawler technology to use reptile depth information to automatically collect geographic data [6]. Cranshaw thought that the potential of location-based (LB) social networks (like Gowalla, Foursquare, and Facebook Places) has already been demonstrated in recent studies and is increasingly exploited as the dimensions of such services grow [7]. Cheng provide an assessment of human mobility patterns by analyzing the spatial, temporal, social, and textual aspects associated with the hundreds of millions of user-driven footprints (i.e., "check-ins") that people leave with these services [8]. Noulas provide a 
similar study but also analyze activity and place transitions [9]. These various studies above have expanded the ideas and methods for POI data collection.

\section{METHODOLOGY AND IMPLEMENTATION}

\section{Methodology}

The overall architecture of the POI data collection scheme based on Google Maps is shown in Figure 1. It consists of a data layer, a service layer, and an application layer.

The data layer is the bottom layer of the entire system and provides data support for the system. The data of the system consists of two basic parts: map data and POI data. The basic map data can be constructed using vector maps and image maps provided by Google Maps, which are called tile maps [10]. The POI data includes nine types of special POI data for schools, hospitals, urban management components, restaurants, tourist attractions, shopping malls, hotels, gas stations, and shelters in Hefei, Anhui Province. These data also include spatial location information and attribute information.

The service layer is located above the data layer and contains multiple types. It can be mainly divided into POI data service, map vector service, map image service. Specifically, the special-purpose POI data service is mainly responsible for the database access of the POI and provides three functions. These functions are the retrieval, insertion, update, and deletion function to the stored POI information data table. According to the request conditions sent by the application layer and the business logic of the system itself, data is read from the POI database, and these data are fed back to the client's request, or the corresponding business process results are stored in the POI database. Vector map service and map image service are mainly used for loading vector map data and image map data from Google map.

The POI application layer is located above the data layer and service layer and is the main client end of the system. The user can obtain the data of interest through the interface and interface provided by the application layer and construct suitable map data. The application layer can build a POI data acquisition system based on Google Maps with the help of the data layer and service layer. This system provides many functions, including basic map operation function, POI information query function, POI result display function, and POI data collection. Function, POI information correction function and POI information supplement function.

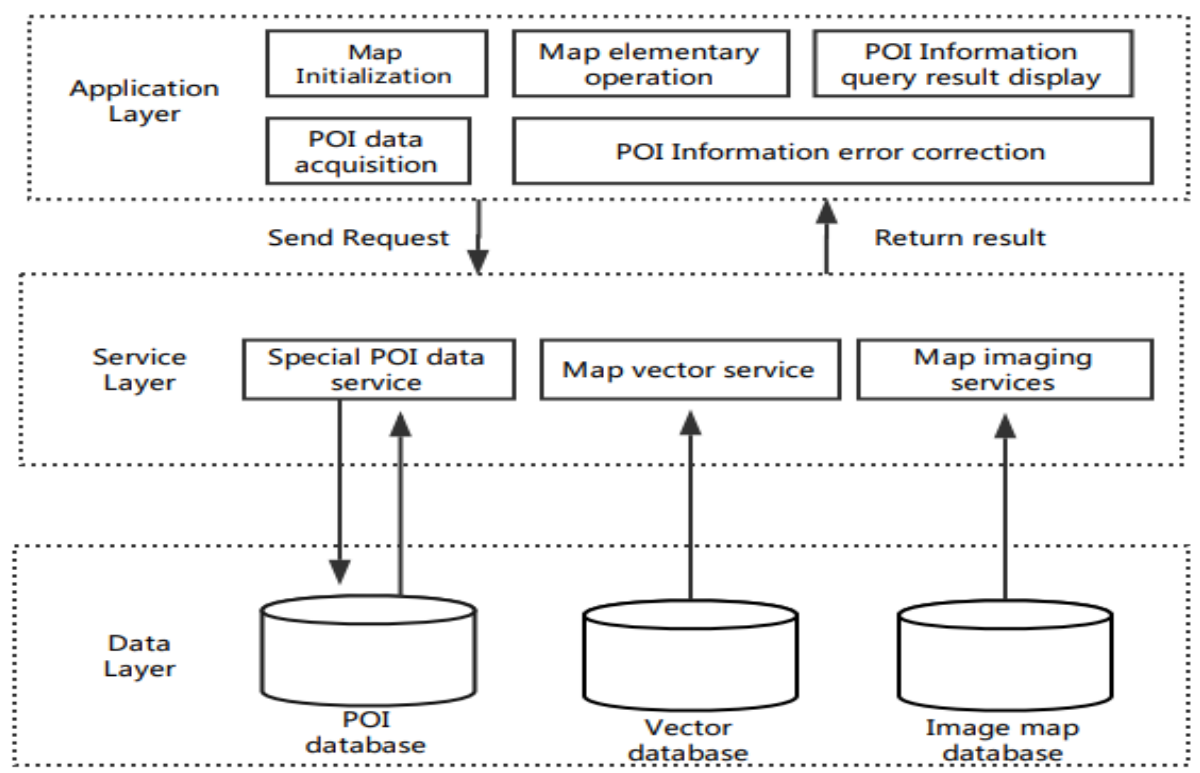

FIGURE 1. POI data acquisition system architecture 
Therefore, in order to complete the POI data collection of the entire system, it needs to be divided into multiple steps. Firstly, the search engine provides a data search service. The user can search through the POI data search engine and query whether there is POI information of interest in the POI database. Then, if the user retrieves the POI information in the POI database, the location of the POI can be superimposed on the map in the form of an annotation using the Google Maps API. If user clicks on the label, the system presents the POI details to the user in the form of an information window. If the user finds that the information is incorrect or the attribute information is incomplete, the user can submit the error reason and supplementary information. The system will review this information in the background and take corresponding measures to ensure the data quality of the POI. If the POI information retrieved in the POI database is not familiar for users, the system will select the thematic layer. The users can mark the position of the POI on the base map, and then dynamically build the attribute collection interface of the POI layer of the topic and enter the attribute information to submit. If the data has already been submitted before, the system will conduct confidential data check, quality inspection and final review. After completing these steps above, the system will confirm the POI information. Confirmation can be based on the mobile device to confirm the POI data on the spot, or through indoor satellite image comparison or telephone survey [11]. After confirmation, the system will merge the confirmed POI data with the existing POI database to build a new POI database.

\section{Implementation}

In order to implement this system, the required development environment is shown in Table 1. After configuring the environment, it is necessary to organize the appropriate data. The basic map data includes vector maps and image maps, all of which are provided by calling vector map services and image map services provided by the map. The map's Web API is written in JavaScript. POI data uses PostGIS in PostgreSQL, an object-relational database, to store POI data. PostgreSQL is an open source database management system that supports rich data types, supports object-oriented technologies, and integrates easily with Web applications. PostGIS is an open source program that provides geospatial data support for PostgreSQL, enabling PostgreSQL to manage spatial data. The system collected the information of schools, hospitals, urban management components, restaurants, tourist attractions, shopping malls, hotels, gas stations, and shelters in Hefei, Anhui Province, and designed a table structure suitable for these data storage.

TABLE 1. System development environment list

\begin{tabular}{|c|c|}
\hline Category & Tool \\
\hline Operating System & Windows 10 \\
\hline IDE & Microsoft Visual Studio 2016, gedit; \\
\hline Web Server & Internet information service manager 6.0 version \\
\hline Language & ASP.Net/C\#, JavaScript, HTML, CSS \\
\hline Database & Postgre SQL/PostGIS \\
\hline Operation Platform & All browsers \\
\hline
\end{tabular}

In order to be able to collect and update POI data online, the system uses the ASP language to write POI data for query, delete, update, add, and other operations and packaged as a special POI data service interface. The front-end development uses JavaScript to call the POI. The data service interface, passing relevant parameters, can operate the POI database, improve the security of the POI database, and reduce the difficulty of the system front-end developer. At the same time, this method can also provide convenience for updating and maintenance of POI data. The functions of the entire system mainly include five parts, which are map vector and image map loading, basic map operations, POI query and display, POI data collection, POI correction and supplement. The system is based on various types of special POI data in Hefei, Anhui Province, for information query and display, and can report errors collected to supplement insufficient information. This scheme can improve the efficiency of urban collection of interest, provide support for urban spatial management, and the advantages and promotion of POI can ensure the smooth progress of smart cities. 


\section{CONCLUSION AND RECOMMENDATION}

The POI database is an important part of the digital city construction and it is also an indispensable support service for geographic information location services. The accuracy, current situation and integrity of POI data have become increasingly important in the rapidly developing technological society. This paper provides a new set of system models based on Google Maps applications and interfaces combined with web development technologies. This system model is based on POI information query and display function of Google Maps. It collects POI data, corrects and supplements the error information, reduces the cost of traditional POI data collection, and improves the efficiency of POI data collection. At the same time, POI data is provided as a service, which makes the update of POI data faster. However, there are still some problems at present, such as the POI classification and classification reminder, the automation of POI data quality inspection and auditing, and the method of matching and fusion based on multi-source POI data will be the next research direction. Due to the rapid development of machine learning and artificial intelligence technologies, it is necessary to focus on how to combine the collection of POI data with these technologies to provide a more intelligent geographic information service system in the future.

\section{REFERENCES}

1. Miyaki, K. (2006). U.S. Patent No. 7,076,741. Washington, DC: U.S. Patent and Trademark Office.

2. Scheibe, B. (2012). U.S. Patent No. 8,165,808. Washington, DC: U.S. Patent and Trademark Office.

3. Goodchild, M. F. (2007). Citizens as voluntary sensors: spatial data infrastructure in the world of Web 2.0. IJSDIR 2: $24-32$.

4. Goodchild M F. Citizens as voluntary sensors: spatial data infrastructure in the world of Web 2.0. IJSDIR 2: 24 - 32[J]. 2007.

5. Li Deren, Qian Xinlin. On the data management of self-disciplined geopolitical information[J]. Wu Han University, 2010.

6. CHEN Ruijia, KANG Zhizhong, ZHANG Weitao. Automatic Acquisition of Navigation Depth Service Information Based on Web Crawler[J]. Surveying and Mapping Engineering, 2015, 24(1): 17-24.

7. Cranshaw J, Schwartz R, Hong J, et al. The livehoods project: Utilizing social media to understand the dynamics of a city[J]. 2012.

8. Cheng Z, Caverlee J, Lee K, et al. Exploring millions of footprints in location sharing services[J]. ICWSM, 2011, 2011: 81-88.

9. Noulas A, Scellato S, Mascolo C, et al. An empirical study of geographic user activity patterns in foursquare[J]. ICwSM, 2011, 11(70-573): 2.

10. Setlur, Vidya, Cynthia Kuo, and Peter Mikelsons. "Towards designing better map interfaces for the mobile: experiences from example." Proceedings of the 1st International Conference and Exhibition on Computing for Geospatial Research \& Application. ACM, 2010.

11. Zhu, Xinyan, and Chunhui Zhou. "POI inquiries and data update based on LBS." Information Engineering and Electronic Commerce, 2009. IEEC'09. International Symposium on. IEEE, 2009. 